\title{
Confronting nuclear equation of state in the presence of dark matter using GW170817 observation in relativistic mean field theory approach
}

\author{
Arpan Das, ${ }^{1, *}$ Tuhin Malik, ${ }^{2, \dagger}$ and Alekha C. Nayak ${ }^{1, \$}$ \\ ${ }^{1}$ Physical Research Laboratory, Ahmedabad 380009, India \\ ${ }^{2}$ Department of Physics, BITS-Pilani, K.K. Birla Goa Campus, Goa 403726, India
}

(Received 2 August 2018; published 28 February 2019)

\begin{abstract}
We confront the admixture of dark matter inside a neutron star using gravitational wave constraints coming from binary neutron star merger. We consider a relativistic mean field model including $\sigma-\omega-\rho$ meson interaction with NL3 parametrization. We study fermionic dark matter interacting with nucleonic matter via Higgs portal mechanism. We show that admixture of dark matter inside the neutron star softens the equation state and lowers the value of tidal deformability. Gravitational wave GW170817 observation puts an upper bound on tidal deformability of a binary neutron star with low spin prior at $90 \%$ confidence level, which disfavors stiff equation of state such as the Walecka model with NL3 parametrization. However, we show that the Walecka model with NL3 parametrization with a fermionic dark matter component satisfies the tidal deformability bound coming from the GW170817 observation.
\end{abstract}

DOI: 10.1103/PhysRevD.99.043016

\section{INTRODUCTION}

Compact objects like neutron stars (NS) are nature's laboratory which can shed light directly or indirectly on the different branches of physics such as low energy nuclear physics, QCD under extreme conditions, the general theory of relativity etc. A neutron star is one of the remnants of a dying star undergoing gravitational collapse. Gravitational collapse of stars with a mass range between 1.4-3.0 $M_{\odot}$ evolve into a neutron star. Neutron degeneracy pressure inside the neutron star makes it hydrostatically stable against the gravitational collapse. If the mass of a dying star is very large (beyond $10 M_{\odot}$ ) then the stellar remnant will overcome the neutron degeneracy pressure and gravitational collapse will produce a black hole. Matter density inside the neutron star can be as large as a few times the nuclear saturation density $\left(n_{B}=0.16 \mathrm{fm}^{-3}\right)$. Interior of a neutron star provides a situation to study the behavior of matter at extreme conditions. In this context, the possible equation of state $(\mathrm{EoS})$ of infinite nuclear matter has been explored extensively (for a brief review see [1]). The main challenge in the description of matter at high densities inside neutron stars is to develop a model which not only

\footnotetext{
*arpan@prl.res.in

†tuhin.malik@gmail.com

*alekha@prl.res.in
}

Published by the American Physical Society under the terms of the Creative Commons Attribution 4.0 International license. Further distribution of this work must maintain attribution to the author(s) and the published article's title, journal citation, and DOI. Funded by SCOAP. describes matter at high densities, but also the properties of matter observed at saturation densities [2-4]. Valid nuclear EoS has to satisfy presently well accepted empirical and experimental constraints [5-7], e.g., ground state properties of spherical and deformed nuclei, saturation density, binding energy, symmetry energy, compression modulus etc. as well as constraints coming from infinite nuclear matter e.g., neutron star mass radius relation, tidal deformability etc. Rotating neutron stars or pulsars give important information about superfluid nature of nucleon inside the neutron star $[8,9]$. Superfluidity of nucleons is important to explain timing irregularities (glitch) of pulsars. From the high energy nuclear physics point of view, neutron star provides an ideal condition where high density QCD matter (quark matter phase, color superconducting phase etc.) can exist [10-13]. Historically the neutron star mass radius relationship coming from solving the Tolman-Oppenheimer-Volkoff (TOV) equation has been studied extensively to put constraints on the nuclear EoS [1]. However recent observation of gravitational waves from neutron star mergers opens up another dimension in the study of the nuclear EoS $[14,15]$.

On August 17, 2017, the Advanced LIGO and Virgo observatories detected the gravitational waves (GW) from a merging binary NS [14]. GW170817 data open up a new way to understand the neutron star structure and the underlying EoS of dense matter. Details of the internal structure of the neutron stars in the binary mergers become important as the orbital separation become comparable with the size of the bodies. For neutron star binary merger the tidal field of the companion induces a quadrupole moment to the other neutron star. The relation of the induced quadrupole moment to the external tidal field is 
proportional to the tidal deformability. Tidal deformability is sensitive to the mass, radius, and tidal love number, which in turn depends on the nuclear EoS. Using observed tidal deformability parameters of the neutron star merger one can put strong constraints on the neutron star EoS, for details and related studies see [16-22] and references therein. The GW170817 observation puts an upper bound on tidal deformability of the combined binary NSs at $90 \%$ confidence [15]. Consequently, this can be used to rule out certain equation of states of neutron stars.

Observations of the kinematics of self-gravitating objects such as galaxies and clusters of galaxies give strong hint of the existence of dark matter (DM). Cosmological observations tell us that this invisible matter cannot consist of baryons, it must be a new kind of matter which interacts with the rest of the standard model particles very weakly. The exact nature of the dark matter, its coupling between standard model particles and the mass is still not known. However extensive studies on the particle physics dark matter models have put strong constraints on the coupling constant and mass of the dark matter particles (for a recent review on dark matter physics see [23]). Among different proposals of dark matter, weakly interacting massive particle (WIMP) scenario has gained favor because it gives the correct prediction of the measured relic abundance of the dark matter today very naturally.

The presence of dark matter inside neutron star and its consequences have been discussed in the literature [24-35]. These discussions include dark matter capture by neutron star and heating of old neutron star in the galactic halo to a temperature detectable by upcoming infrared telescopes [24], trapped WIMPs inside the neutron star [25], charged massive dark matter particle and its effect on neutron star [26], heating of a neutron star due to dark matter annihilation [27-29], or the collapse of a neutron star due to accretion of nonannihilating dark matter [30] etc. In Ref. [32] authors have considered possible effects of a self-interacting dark matter core on the maximum mass of a neutron star, mass-radius relation and on the NS tidal deformability parameter. They have computed radial density and pressure profiles of the baryonic and dark matter components for different nuclear EoS and different dark matter fractions. In Ref. [35], the authors have considered the Walecka relativistic mean field model including $\sigma-\omega$ interaction for the nucleonic part $[2,3,5,6]$ and fermionic dark matter inside the neutron star. Using mean field approximation they have calculated effective nucleon mass, variation of $\sigma$ field, EoS and the corresponding mass radius relation in this model. However, it is important to mention that the simple relativistic mean field model (RMF) model taken in this work is unsuccessful in producing nuclear saturation properties. This simple model is ruled out due to the fact that it gives high nuclear incompressibility $(\approx 500 \mathrm{MeV})$ and very low nucleon mass [36]. Keeping this limitation of the simple $\sigma-\omega$ in this work we have considered the a generalized Lagrangian for the nucleonic sector including $\sigma-\omega-\rho$ meson interaction with NL3 parametrization $[37,38]$. The EoS of this model is disfavored by GW170817 tidal deformability upper bound limit. However, we show that if we consider fermionic dark matter interaction via Higgs portal mechanism, then it can evade the GWs tidal deformability upper bound constraint. We have also considered nongravitational interaction of the dark matter and the nucleon field in a relativistic mean field approach.

This paper is organized as follows: in Sec. II we discuss generalized Walecka model with NL3 parameterization. In Sec. III, we discuss fermionic dark matter model and its interaction with nuclear matter. The EoS of total Lagrangian density of dark matter and nuclear matter is presented in Sec. IV. Constraint from GWs tidal deformability on EoS is discussed in Sec. V. Finally, we conclude in Sec. VI.

\section{WALECKA MODEL WITH NL3 PARAMETRIZATION}

In this section, we briefly summarize the taken RMF model $[2,3,5,6]$, which is also known as quantum hadron dynamics (QHD) [39]. In this framework, nucleons are quasiparticles with an effective medium dependent mass and baryon chemical potential. They move in the mean field of mesons. The simplest QHD model is known as the $\sigma-\omega$ model. In this model nucleon-nucleon interaction is mediated by the exchange of $\sigma$ and $\omega$ mesons. Properties of symmetric nuclear matter has been studied in this model. In general $\sigma$ mesons give rise to a strong attractive central force and a spin-orbit nuclear force, on the other hand, $\omega$ mesons are responsible for the repulsive central force. However, this simple model does not reproduce nuclear saturation properties, e.g., compressibility [36]. More advanced versions of QHD includes $\rho$ meson exchange interaction between nucleons [37]. Since protons and neutrons only differ in terms of their isospin projections, $\rho$ mesons are included to distinguish between these baryons and to give a better account of the symmetry energy. These vector mesons are charged, hence the reaction between $\rho$ meson and proton will differ from the reaction between $\rho$ meson and neutron. In general one can also include photon field, however, neutron star is assumed to be charge neutral, hence the contribution from the photon field can be neglected.

Lagrangian including nucleon field, $\sigma, \omega$ and $\rho$ mesons and their interactions can be written as [37],

$$
\begin{aligned}
\mathcal{L}= & \bar{\psi}\left[\gamma^{\mu}\left(i \partial_{\mu}-g_{v} V_{\mu}-g_{\rho} \boldsymbol{\tau} \cdot \boldsymbol{b}_{\boldsymbol{\mu}}\right)-\left(M_{n}+g_{s} \boldsymbol{\phi}\right)\right] \psi \\
& +\frac{1}{2} \partial_{\mu} \phi \partial^{\mu} \phi-\frac{1}{2} m_{s}^{2} \phi^{2}-\frac{1}{3} g_{2} \phi^{3}-\frac{1}{4} g_{3} \phi^{4} \\
& -\frac{1}{4} V^{\mu \nu} V_{\mu \nu}+\frac{1}{2} m_{V}^{2} V^{\mu} V_{\mu}-\frac{1}{4} \boldsymbol{b}^{\mu \nu} \cdot \boldsymbol{b}_{\mu \nu}+\frac{1}{2} m_{\rho}^{2} \boldsymbol{b}^{\mu} \boldsymbol{b}_{\boldsymbol{\mu}}
\end{aligned}
$$


In general one can also include terms quartic in $\omega$ meson and $\omega-\rho$ interactions. However in the present work we have used NL3 parametrization of the above Lagrangian. In this parametrization coupling of terms quartic in $\omega$ meson and $\omega-\rho$ interactions are taken to be zero. In the above equation $\psi$ is nucleon doublet, $\phi, V_{\mu}$, and $\boldsymbol{b}_{\mu}$ denotes $\sigma, \omega, \rho$ meson field, respectively. $m_{s}, m_{V}$, and $m_{\rho}$ are the masses of the mesons and $M_{n}$ denotes the nucleon mass. $g_{s}, g_{v}$, and $g_{\rho}$ are the scalar, vector, and isovector coupling constants, respectively. Field strength tensor of the vector and isovector mesons are given by,

$$
V_{\mu \nu}=\partial_{\mu} V_{\nu}-\partial_{\nu} V_{\mu},
$$

and,

$$
\boldsymbol{b}_{\mu \nu}=\partial_{\mu} \boldsymbol{b}_{\nu}-\partial_{\nu} \boldsymbol{b}_{\mu}
$$

$\rho$ meson field can be written explicitly as, $\boldsymbol{b}^{\mu}=$ $\left(b_{1}^{\mu}, b_{2}^{\mu}, b_{3}^{\mu}\right) . b_{3}^{\mu}$ represents neutral $\rho^{0}$ meson and $\rho^{ \pm}$are the orthogonal linear superposition of $b_{1}^{\mu}$ and $b_{2}^{\mu}$.

$$
b_{ \pm}^{\mu}=\frac{1}{\sqrt{2}}\left(b_{1}^{\mu} \pm b_{2}^{\mu}\right) \text {. }
$$

$\boldsymbol{\tau}=\left(\tau_{1}, \tau_{2}, \tau_{3}\right)$ are the Pauli matrices, these are also the isospin operators. Proton and neutron are the different projections of nucleon in isospin space. Operation of $\tau_{3}$ on neutron and proton is as follows,

$$
\tau_{3}|p\rangle=+1|p\rangle \quad \text { and } \quad \tau_{3}|n\rangle=-1|n\rangle
$$

Numerical values of the all the parameter of the Lagrangian are given in the Table I [37].

\section{INTERACTION LAGRANGIAN BETWEEN NUCLEAR MATTER AND DARK MATTER}

Due to the galaxy rotation, the compact object like neutron star pass through the dark matter halo and capture dark matter particle from it. Because of the high baryon density inside neutron star, the DM particle loses energy due to its interaction with neutrons. The strong gravitational force of the NS trap the DM after it loses some energy $[27,34,40]$. There are also other mechanism such as conversion neutrons to scalar dark matter, scalar DM production via bremsstrahlung increases the dark matter density inside the neutron star $[32,33]$. Since dark matter composed of $95 \%$ total matter density, one could possible imagine that many compact objects composed of DM. The amount of dark matter inside NS also depend on the evolution history of NS, the environment where it lives. In Ref. [41], the authors have shown that the binary neutron star systems might enhance DM accumulation probability inside NS.
We consider fermionic dark matter $(\chi)$ inside the neutron star. Here we consider the lightest neutralino which acts as a fermionic dark matter candidate [35,42]. Dark matter is not directly coupled with the nucleons rather they are coupled to the Higgs field $h$. Coupling between the dark matter and the Higgs field is $y$. For neutralino mass $\left(M_{\chi}=200 \mathrm{GeV}\right)$, the value of $y$ varies from $0.001-0.1$. We fix $y=0.07$ for the rest of our analysis [35,42]. The Higgs field is also coupled to the nucleons through effective Yukawa coupling $\frac{f M_{n}}{v}$, where $f$ the proton-Higgs form factor and its value has been estimated to be approximately 0.35 [43]. We have not considered $h^{3}$ and $h^{4}$ term in the Higgs potential, because in the mean field approximation the value of the $h$ is small and the only dominant term is the $h^{2}$. So, the dark sector Lagrangian and its interaction with the nucleons and Higgs field is given by [35]

$$
\begin{aligned}
\mathcal{L}_{\mathrm{DM}}= & \bar{\chi}\left[i \gamma^{\mu} \partial_{\mu}-M_{\chi}+y h\right] \chi+\frac{1}{2} \partial_{\mu} h \partial^{\mu} h-\frac{1}{2} M_{h}^{2} h^{2} \\
& +f \frac{M_{n}}{v} \bar{\psi} h \psi .
\end{aligned}
$$

Direct detection experiment such as LUX [44] and XENON [45] excluded dark matter-nucleon cross section above $\sim 8 \times 10^{-47} \mathrm{~cm}^{2}$ for dark matter mass range 30$50 \mathrm{GeV}$ at $90 \%$ C.L.. The invisible Higgs decay width tightly constraint the dark matter below $M_{h} / 2$, hence the dark matter mass $m_{\chi}=200 \mathrm{GeV}$ evades these constraints. It is important to mention that dark matter may not be a single component, it may well be a multicomponent system. Dark matter can consist of low mass as well as high mass particles. As an example in Ref. [35] authors have considered heavy dark matter particles inside the neutron star.

Here we have considered the average number density of nuclear matter is $10^{3}$ times larger than the average dark matter density $\left(n_{\mathrm{DM}}\right)$, which gives the ratio between mass of the dark matter inside neutron star and mass of the neutron star to be $\sim \frac{1}{6}$ [35]. We know that nuclear saturation density $n_{B} \sim 0.16 \mathrm{fm}^{-3}$, so dark matter number density is $n_{\mathrm{DM}} \sim 10^{-3} n_{B} \sim 0.16 \times 10^{-3} \mathrm{fm}^{-3}$. Number density of dark matter $n_{\mathrm{DM}}=\frac{\left(k_{F}^{\mathrm{DM}}\right)^{3}}{3 \pi^{2}}$, which gives $k_{F}^{\mathrm{DM}} \sim 0.033 \mathrm{GeV}$. In our calculations we have varied $k_{F}^{\mathrm{DM}}$ from $0.02 \mathrm{GeV}$ to $0.06 \mathrm{GeV}$. For these values of $k_{F}^{\mathrm{DM}}$ corresponding dark matter density will be different.

\section{NEUTRON STAR EQUATION OF STATE AND BETA EQUILIBRIUM}

The Euler Lagrange equation of motion for nucleon doublet $(\psi)$, scalar $(\phi)$, vector $\left(V^{\mu}\right)$, isovector $\left(\boldsymbol{b}^{\mu}\right)$, DM particle $(\chi)$ and Higgs boson $(h)$ can be derived from Lagrangian densities Eqs. (1) and (6) as, 
TABLE I. Nucleon masses $\left(M_{n}\right), \sigma$ meson mass $\left(m_{s}\right), \omega$ meson mass $\left(m_{v}\right), \rho$ mass $m_{\rho}$ and couplings $g_{s}, g_{v}, g_{\rho}, g_{2}, g_{3}$ of NL3 parametrization [37].

\begin{tabular}{lcccccccc}
\hline \hline$M_{n}(\mathrm{MeV})$ & $m_{s}(\mathrm{MeV})$ & $m_{v}(\mathrm{MeV})$ & $m_{\rho}(\mathrm{MeV})$ & $g_{s}$ & $g_{v}$ & $g_{\rho}$ & $g_{2}\left(\mathrm{fm}^{-1}\right)$ & $g_{3}$ \\
\hline 939 & 508.194 & 782.501 & 763.000 & 10.217 & 12.868 & 4.474 & -10.431 & -28.885 \\
\hline \hline
\end{tabular}

$\left[\gamma^{\mu}\left(i \partial_{\mu}-g_{v} V_{\mu}-g_{\rho} \boldsymbol{\tau} \cdot \boldsymbol{b}_{\mu}\right)-\left(M_{n}+g_{s} \boldsymbol{\phi}-\frac{f M_{n}}{v} h\right)\right] \psi=0$,

$\partial_{\mu} \partial^{\mu} \phi+m_{s}^{2} \phi+g_{2} \phi^{2}+g_{3} \phi^{3}+g_{s} \bar{\psi} \psi=0$,

$\partial_{\mu} V^{\mu \nu}+m_{V}^{2} V^{\nu}=g_{v} \bar{\psi} \gamma^{\nu} \psi$

$\partial_{\mu} \boldsymbol{b}^{\mu \nu}+m_{\rho}^{2} \boldsymbol{b}^{\nu}=g_{\rho} \bar{\psi} \gamma^{\nu} \boldsymbol{\tau} \psi$

$\left(i \gamma_{\mu} \partial^{\mu}-M_{\chi}+y h\right) \chi=0$,

$\partial_{\mu} \partial^{\mu} h+M_{h}^{2} h=y \bar{\chi} \chi+\frac{f M_{n}}{v} \bar{\psi} \psi$,

respectively. The DM particle mass and Higgs particle mass are denoted as $M_{\chi}$ and $M_{h}$, respectively. Applying standard relativistic mean field approximation we get,

$$
\begin{aligned}
\phi_{0}= & \frac{1}{m_{s}^{2}}\left(-g_{s}\langle\bar{\psi} \psi\rangle-g_{2} \phi_{0}^{2}-g_{3} \phi_{0}^{3}\right), \\
V_{0}= & \frac{g_{v}}{m_{V}^{2}}\left\langle\psi^{\dagger} \psi\right\rangle=\frac{g_{v}}{m_{V}^{2}}\left(\rho_{p}+\rho_{n}\right), \\
h_{0}= & \frac{y\langle\bar{\chi} \chi\rangle+f \frac{M_{n}}{v}\langle\bar{\psi} \psi\rangle}{M_{h}^{2}}, \\
b_{0}= & \frac{g_{\rho}}{M_{\rho}^{2}}\left\langle\psi^{\dagger} \tau_{3} \psi\right\rangle=\frac{g_{\rho}}{M_{\rho}^{2}}\left(\rho_{p}-\rho_{n}\right), \\
& \left(i \gamma^{\mu} \partial_{\mu}-g_{v} \gamma^{0} V_{0}-g_{\rho} \tau_{3} \gamma^{0} b_{0}-M_{n}^{\star}\right) \psi=0, \\
& \left(i \gamma^{\mu} \partial_{\mu}-M_{\chi}^{\star}\right) \chi=0,
\end{aligned}
$$

where $M_{n}^{\star}$ and $M_{\chi}^{\star}$ are nucleon and dark matter effective mass, respectively. $\rho_{p}$ and $\rho_{n}$ are the densities of proton and neutron, respectively. The effective mass of nucleon and dark matter can be given as,

$$
\begin{aligned}
& M_{n}^{\star}=M_{n}+g_{s} \phi_{0}-\frac{f M_{n}}{v} h_{0}, \\
& M_{\chi}^{\star}=M_{\chi}-y h_{0} .
\end{aligned}
$$

The baryon density $(\rho)$, scalar density $\left(\rho_{s}\right)$, and dark matter scalar density $\left(\rho_{s}^{\mathrm{DM}}\right)$ are

$$
\begin{aligned}
\rho & =\left\langle\psi^{\dagger} \psi\right\rangle=\frac{\gamma}{(2 \pi)^{3}} \int_{0}^{k_{F}} d^{3} k, \\
\rho_{s} & =\langle\bar{\psi} \psi\rangle=\frac{\gamma}{(2 \pi)^{3}} \int_{0}^{k_{F}} \frac{M_{n}^{\star}}{\sqrt{M_{n}^{\star 2}+k^{2}}} d^{3} k, \\
\rho_{s}^{\mathrm{DM}} & =\langle\bar{\chi} \chi\rangle=\frac{\gamma}{(2 \pi)^{3}} \int_{0}^{k_{F}^{\mathrm{DM}}} \frac{M_{\chi}^{\star}}{\sqrt{M_{\chi}^{\star 2}+k^{2}}} d^{3} k,
\end{aligned}
$$

where $k_{F}$ and $k_{F}^{\mathrm{DM}}$ are the Fermi momentum for the nucleonic matter and dark matter respectively. $\gamma$ is the spin degeneracy factor of nucleon and $\gamma=2$ for neutron and proton individually.

The masses for both nucleon and dark matter depends on baryon density for fixed values of dark matter Fermi momentum $k_{F}^{\mathrm{DM}}$ and coupling constants. These masses and coupling values are discussed in Table I and Sec. III. To get the density dependent profile for $M_{n}^{\star}$ and $M_{\chi}^{\star}$, one needs to solve numerically Eq. (10) together with the field equations Eq. (8) in self-consistent manner. In this work, we have taken the average dark matter number density approximately 1000 times smaller than the average neutron number density. This implies the dark matter mass fraction with respect to the neutron star mass is $\simeq 1 / 6$. The expectation values of the energy-momentum tensor or the stress tensor provide the energy density and pressure of the system in the static case, i.e., the EoS, which is given by,

$$
\epsilon=\left\langle T^{00}\right\rangle \quad \text { and } \quad P=\frac{1}{3}\left\langle T^{i i}\right\rangle .
$$

The expression for the total energy density $(\epsilon)$ and the pressure $(P)$ can be obtained by combining the Lagrangian density Eqs. (1) and (6):

$$
\begin{aligned}
& \epsilon=g_{v} V_{0}\left(\rho_{p}+\rho_{n}\right)+g_{\rho} b_{0}\left(\rho_{p}-\rho_{n}\right)+\frac{1}{\pi^{2}} \int_{0}^{k_{p}} d k k^{2} \sqrt{k^{2}+\left(M_{n}^{\star}\right)^{2}}+\frac{1}{\pi^{2}} \int_{0}^{k_{n}} d k k^{2} \sqrt{k^{2}+\left(M_{n}^{\star}\right)^{2}}+\frac{1}{\pi^{2}} \int_{0}^{k_{F}^{\mathrm{DM}}} d k k^{2} \sqrt{k^{2}+\left(M_{\chi}^{\star}\right)^{2}} \\
& +\frac{1}{2} m_{s}^{2} \phi_{0}^{2}+\frac{1}{3} g_{2} \phi_{0}^{3}+\frac{1}{4} g_{3} \phi_{0}^{4}-\frac{1}{2} m_{V}^{2} V_{0}^{2}-\frac{1}{2} m_{\rho}^{2} b_{0}^{2}+\frac{1}{2} M_{h} h_{0}^{2} . \\
& P=\frac{1}{3 \pi^{2}} \int_{0}^{k_{p}} \frac{k^{4} d k}{\sqrt{k^{2}+\left(M_{n}^{\star}\right)^{2}}}+\frac{1}{3 \pi^{2}} \int_{0}^{k_{n}} \frac{k^{4} d k}{\sqrt{k^{2}+\left(M_{n}^{\star}\right)^{2}}}+\frac{1}{3 \pi^{2}} \int_{0}^{k_{F}^{\text {DM }}} \frac{k^{4} d k}{\sqrt{k^{2}+\left(M_{\chi}^{\star}\right)^{2}}} \\
& -\frac{1}{2} m_{s}^{2} \phi_{0}^{2}-\frac{1}{3} g_{2} \phi_{0}^{3}-\frac{1}{4} g_{3} \phi_{0}^{4}+\frac{1}{2} m_{V}^{2} V_{0}^{2}+\frac{1}{2} m_{\rho}^{2} b_{0}^{2}-\frac{1}{2} M_{h} h_{0}^{2}
\end{aligned}
$$


$\rho_{n}$ and $\rho_{p}$ are the neutron and proton number density with $k_{F}^{n}$ and $k_{F}^{p}$ are the corresponding Fermi momentum of neutron and proton, respectively. The number densities and corresponding Fermi momenta are equal for the symmetric nuclear matter. The matter inside the neutron star is mainly composed of neutrons. However, the neutron will eventually $\beta$ - decays as,

$$
\begin{aligned}
& n \rightarrow p+e^{-}+\bar{\nu}, \\
& n+\nu \rightarrow p+e^{-} .
\end{aligned}
$$

To maintain the neutron star matter charge neutral, muons $(\mu)$ will appear when the chemical potential of the electrons reaches the muon rest mass $\left(m_{\mu}=106 \mathrm{MeV}\right)$. For a given baryon density $\left(\rho=\rho_{n}+\rho_{p}\right)$, the charge neutrality is given as,

$$
\rho_{p}=\rho_{e}+\rho_{\mu}
$$

and the $\beta$ - equilibrium condition is given as,

$$
\mu_{n}=\mu_{p}+\mu_{e} \quad \text { and } \quad \mu_{e}=\mu_{\mu}
$$

Where, the chemical potentials $\mu_{p}, \mu_{n}, \mu_{e}$, and $\mu_{\mu}$ are given as,

$$
\begin{gathered}
\mu_{p}=\frac{\partial \epsilon}{\partial \rho_{p}}=g_{v} V_{0}+g_{\rho} b_{0}+\sqrt{k_{p}^{2}+\left(M_{n}^{\star}\right)^{2}} \\
\mu_{n}=\frac{\partial \epsilon}{\partial \rho_{n}}=g_{v} V_{0}-g_{\rho} b_{0}+\sqrt{k_{n}^{2}+\left(M_{n}^{\star}\right)^{2}} \\
\mu_{e}=\sqrt{k_{e}^{2}+m_{e}^{2}} \\
\mu_{\mu}=\sqrt{k_{\mu}^{2}+m_{\mu}^{2}}
\end{gathered}
$$

The particle fractions of neutrons and protons will depend on both charge neutrality and the $\beta$ - equilibrium condition as given above. The self-consistent numerical solution of Eqs. (16) and (17) will set the fraction of neutron, proton, electron, and muon number density for a given baryon density. The total energy density and pressure of leptons are given as,

$$
\begin{gathered}
\epsilon_{l}=\sum_{l=e, \mu} \frac{1}{\pi^{2}} \int_{0}^{k_{l}} k^{2} \sqrt{k^{2}+m_{l}^{2}} d k \\
P_{l}=\sum_{l=e, \mu} \frac{1}{3 \pi^{2}} \int_{0}^{k_{l}} \frac{k^{4} d k}{\sqrt{k^{2}+m_{l}^{2}}}
\end{gathered}
$$

The total energy density and pressure for $\beta$ - equilibrated neutron star matter are

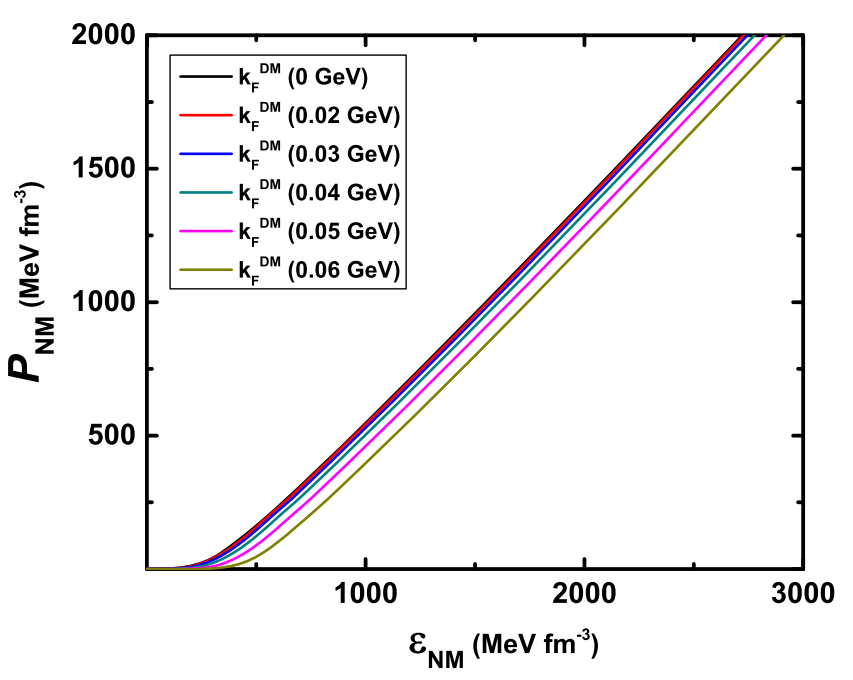

FIG. 1. The equation of states of NS with different dark matter Fermi momenta $k_{F}^{\mathrm{DM}}, 0.0-0.06 \mathrm{GeV}$ with a step of $0.02 \mathrm{GeV}$. The black line corresponds to EoS of NS without dark matter. The EoS becomes softer with increasing number density of dark matter inside the NS.

$$
\begin{gathered}
\epsilon_{\mathrm{NM}}=\epsilon_{l}+\epsilon, \\
P_{\mathrm{NM}}=P_{l}+P .
\end{gathered}
$$

In Fig. 1, we plot pressure $\left(P_{\mathrm{NM}}\right)$ as function of the total energy density $\left(\epsilon_{\mathrm{NM}}\right)$ for different dark matter Fermi momentum $\quad k_{F}^{\mathrm{DM}}=0.0-0.06 \mathrm{GeV}$. Fermi momentum $k_{F}^{\mathrm{DM}}=0.0 \mathrm{GeV}$ corresponds equation of state without dark matter. Increasing the value of $k_{F}^{\mathrm{DM}}$ from $0.02 \mathrm{GeV}$ to $0.06 \mathrm{GeV}$, the EoS becomes softer, i.e., with increasing density of dark matter pressure reduces, which is consistent with earlier work [35]. This behavior is evident from the expression of energy density and pressure from Eqs. (12) and (13), i.e., with increasing $k_{F}^{\mathrm{DM}}$ the dark matter contribution in energy density increases much faster than the pressure.

Neutron star mass radius relationship can be obtained by solving the TOV equation for a given nuclear matter EoS [46]. The EoS for the core is obtained from the Walecka Model with NL3 parameterization in the presence and absence of dark matter components. Crust EoS is modeled using the BPS EoS [47] for the range of density $\rho \sim 4.8 \times$ $10^{-9}$ to $2.6 \times 10^{-4} \mathrm{fm}^{-3}$. We use the polytropic form $P_{\mathrm{NM}}\left(\epsilon_{\mathrm{NM}}\right)=a_{1}+a_{2} \epsilon_{\mathrm{NM}}^{\gamma^{\prime}}$ [48] to join the core and crust of the NS, where $a_{1}$ and $a_{2}$ are obtained by matching the edge of the core at one end with the inner edge of the outer crust at other end, and $\gamma^{\prime}$ is taken 4/3 [49]. In Fig. 2 we plot the mass radius of NS using the EoS as shown in Fig. 1. It is clear from Fig. 1 that the equation state is softer in the presence of larger dark matter density. A softer equation of state gives a lower value of the maximum mass of neutron star. From Fig. 2, the maximum mass without dark matter 


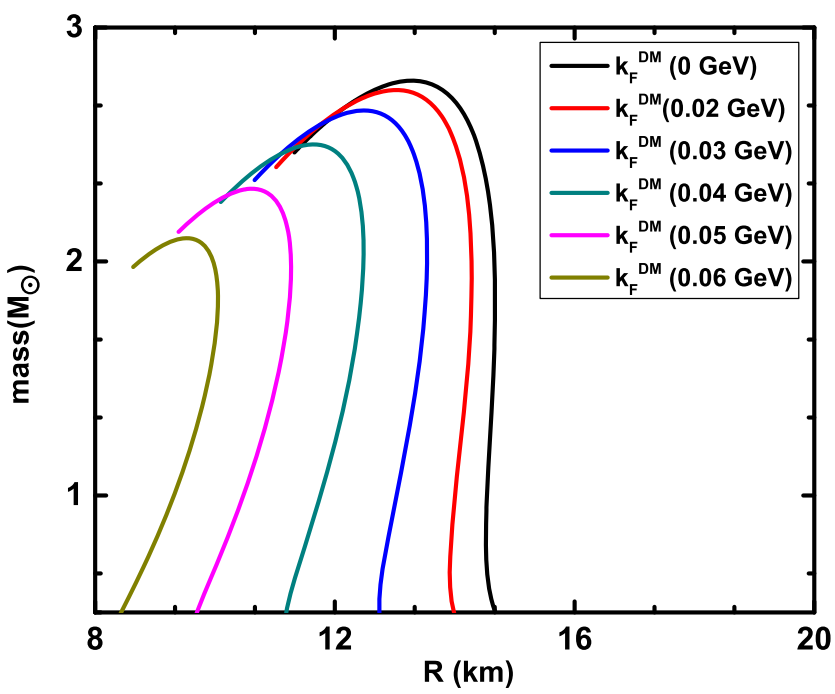

FIG. 2. Mass radius relation of NS for different EoS with dark matter Fermi momentum $k_{F}^{\mathrm{DM}}=0.0-0.06 \mathrm{GeV}$. The maximum mass of NS without dark matter is $2.8 M_{\odot}$ and $2.1 M_{\odot}$ with dark matter Fermi momentum $k_{F}^{\mathrm{DM}}=0.06 \mathrm{GeV}$.

$\left(k_{F}^{\mathrm{DM}}=0\right) \sim 2.8 M_{\odot}$ and the value of the maximum mass of NS decreases with increasing dark matter density.

In the context of dark matter inside the neutron star it is very natural to consider the formation of dark matter corelike structure inside the neutron star with nonuniform distribution of the dark matter. This picture has been explored in various literatures [32,34]. We would like to point out that we are focusing on the mainly qualitative aspect of the presence of dark matter inside the neutron star. The effect of nonuniformly distributed dark matter inside the neutron star on its properties has been discussed in Ref. [34]. In Ref. [34] authors have invoked two-fluid picture of the neutron star containing nuclear matter fluid as well as dark matter fluid inside the neutron star. In that work, authors also have considered the RMF picture for both nuclear fluid as well as dark matter fluid, the mass radius relation in the presence of dark matter for some specific EOS is qualitatively similar to our result, e.g., in the presence of dark matter, mass of neutron star can decrease. They have also shown that for mass of the dark matter $\left(M_{\mathrm{DM}}\right)$ can be large e.g., $0.33 M_{\odot}$. However, if one assumes a RMF kind of situation for the dark matter sector then the coupling of the dark matter particle with a scalar, vector particles etc. will be free parameters of the theory. In the nuclear matter sector, the coupling constants have been fixed keeping in mind the finite nuclei properties, but for the dark matter, these constraints are not available. Hence these free coupling constants in the dark matter sector will make the model less predictive.

Tidal deformability also depends upon the compactness and equation of state. In the next section, we study the effect of dark matter on nuclear matter EoS using the tidal deformability constraint from GW170817 observation.

\section{TIDAL DEFORMABILITY CONSTRAINT}

One of the important measurable structural properties of a binary merger is the tidal deformability. In a coalescing binary NS system, during the last stage of inspiral, each NS develops a mass quadrupole due to the tidal gravitational field induced by the other NS forming the binary. The tidal deformability describes the degree of deformation of a NS due to the tidal field of the companion NS and is sensitive to the nature of the EoS. The tidal deformability is defined as,

$$
\lambda=\frac{2}{3} k_{2} R^{5},
$$

where $R$ is the radius of the NS. The value of $k_{2}$ is typically in the range $\simeq 0.05-0.15$ [16-18] for NSs and depends on the stellar structure. This quantity can be calculated using the following expression [16]

$$
\begin{aligned}
k_{2}= & \frac{8 C^{5}}{5}(1-2 C)^{2}\left[2+2 C\left(y_{R}-1\right)-y_{R}\right] \\
& \times\left\{2 C\left(6-3 y_{R}+3 C\left(5 y_{R}-8\right)\right)\right. \\
& +4 C^{3}\left[13-11 y_{R}+C\left(3 y_{R}-2\right)+2 C^{2}\left(1+y_{R}\right)\right] \\
& \left.+3(1-2 C)^{2}\left[2-y_{R}+2 C\left(y_{R}-1\right)\right] \log (1-2 C)\right\}^{-1},
\end{aligned}
$$

where $C(\equiv M / R)$ is the compactness parameter of the star of mass $M$. The quantity $y_{R}(\equiv y(R))$ can be obtained by solving the following differential equation

$$
r \frac{d y(r)}{d r}+y(r)^{2}+y(r) F(r)+r^{2} Q(r)=0
$$

with

$$
\begin{aligned}
F(r)= & \frac{r-4 \pi r^{3}(\epsilon(r)-p(r))}{r-2 M(r)}, \\
Q(r)= & \frac{4 \pi r\left(5 \epsilon(r)+9 p(r)+\frac{\epsilon(r)+p(r)}{\partial p(r) / \partial \epsilon(r)}-\frac{6}{4 \pi r^{2}}\right)}{r-2 M(r)} \\
& -4\left[\frac{M(r)+4 \pi r^{3} p(r)}{r^{2}(1-2 M(r) / r)}\right]^{2},
\end{aligned}
$$

along with the TOV equation with proper boundary conditions $[46,50]$. One can then define the dimensionless tidal deformability: $\Lambda=\frac{2}{3} k_{2} C^{-5}$.

Individual dimensionless tidal deformability of two stars, $\Lambda_{1}$ and $\Lambda_{2}$ cannot be extracted independently from the observed gravitational waveform. Instead, an effective dimensionless tidal deformability of the binary $\tilde{\Lambda}$ can be extracted, which is a mass-weighted average of the individual dimensionless tidal deformability $\Lambda_{1}$ and $\Lambda_{2}$. The effective tidal deformability $(\tilde{\Lambda})$ of binary system in terms of $\Lambda_{1}$ and $\Lambda_{2}$ is defined as [51] 

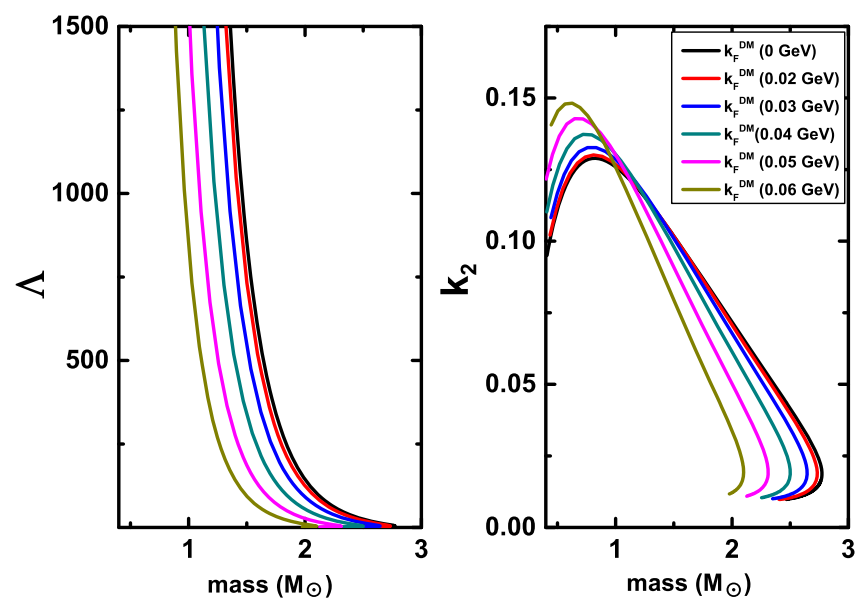

FIG. 3. Dimensionless tidal deformability $(\Lambda)$ of NS and Love number $\left(k_{2}\right)$ as a function of neutron star mass for different dark matter Fermi momentum is shown.

$\tilde{\Lambda}=\frac{16}{13} \frac{\left(m_{1}+12 m_{2}\right) m_{1}^{4} \Lambda_{1}+\left(m_{2}+12 m_{1}\right) m_{2}^{4} \Lambda_{2}}{\left(m_{1}+m_{2}\right)^{5}}$

where $m_{1}$ and $m_{2}$ are the masses of the neutron stars in the binary. Similarly, masses of the two companion neutron stars cannot be measured directly, rather the chirp mass, $\mathcal{M}_{c}=\left(m_{1} m_{2}\right)^{3 / 5}\left(m_{1}+m_{2}\right)^{-1 / 5}$, which is measured directly. By assuming low-spin prior which is consistent with the binary neutron star systems that have been observed in, GW170817 put an upper bound on the NSs combined dimensionless tidal deformability and chirp mass with $90 \%$ confidence [15]. This analysis predicts that the combined dimensionless tidal deformability of the NS merger is $\tilde{\Lambda} \leq 800$. It is important to note that a reanalysis of GW170817 observation has been done assuming the same EoS for both stars and this puts an upper limit on the dimensionless tidal deformability, $\tilde{\Lambda} \leq 1000$ [52]. However lower bound on dimensionless tidal deformability can be put using the investigation of the UV/optical/ infrared counterpart of GW170817 with kilonova models [53]. The lower bound of dimensionless tidal deformability is $\tilde{\Lambda} \geq 400$.

One of the important goals of this work is to study the structural properties of neutron stars in the presence of the dark matter component. For the sake of arguments it is important to understand the behavior of dimensionless tidal deformability and the tidal Love number of the companion neutron stars. Solving Eq. (28) and the TOV equation with appropriate boundary conditions, we get $y_{R}$. Using the value of $y_{R}$ and compactness one can get $k_{2}$ using the expression Eq. (27). Left plot in Fig. 3 shows the dimensionless tidal deformability $\Lambda$ and the right plot shows tidal Love number $k_{2}$ as a function of the NS mass for our EoS with different dark matter density. The value of $k_{2}$ is of the range 0.09-0.13 for a typical neutron star of mass $1.5 M_{\odot}$, which is expected [16,17]. For a given

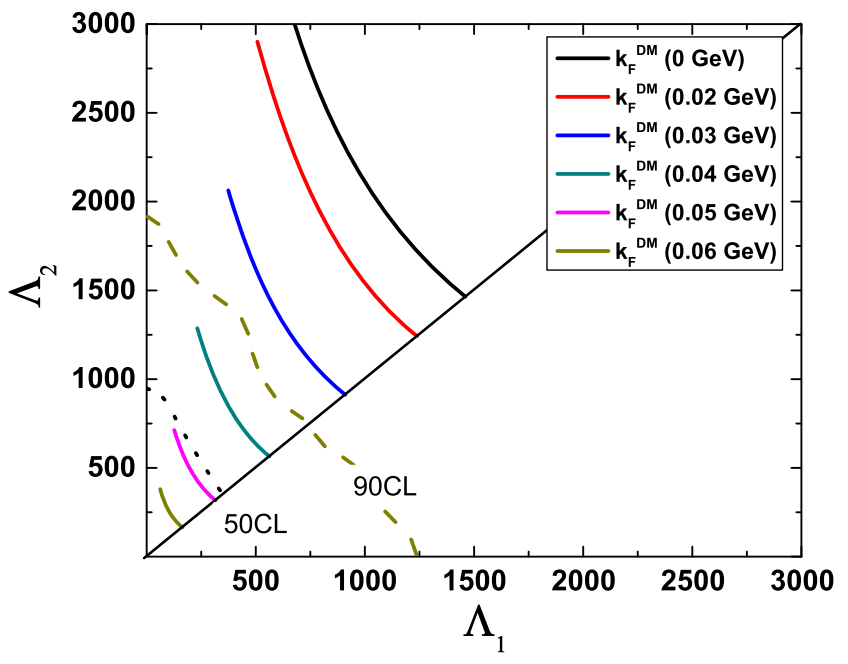

FIG. 4. Tidal deformability parameters of the low and high mass components of binary neutron star merger (GW170817 observation). Dashed line and dotted line indicates $90 \%$ and $50 \%$ confidence limit for low spin priors [15]. The diagonal solid line corresponds to $\Lambda_{1}=\Lambda_{2}$ boundary. The Walecka model with NL3 parametrization is disfavored by GW observation at $90 \%$ C.L. in the absence of dark matter, i.e., $k_{F}^{\mathrm{DM}}=0.0 \mathrm{GeV}$. Note that low dark matter density, e.g., $k_{F}^{\mathrm{DM}}=0.03 \mathrm{GeV}$ is also disfavored. However, the Walecka model in NL3 parametrization with relatively higher DM density, e.g., $k_{F}^{\mathrm{DM}}=0.04-0.06 \mathrm{GeV}$ is allowed by $90 \%$ C.L. of the GW170817 observation.

neutron star mass (say around $1.5 M_{\odot}$ ) EoS without the dark matter predicts larger radius and with increasing dark matter density radius decreases. Since the dimensionless tidal deformability is inversely proportional to the compactness $(C=M / R)$, its value is larger in the absence of dark matter.

To study the tidal deformability constraint from the GW170817 observation on EoS of NS, we plot the combined tidal deformability of the binary system in $\Lambda_{1}$, $\Lambda_{2}$ plane in Fig. $4 . \Lambda_{1}$ and $\Lambda_{2}$ are the individual dimensionless tidal deformability of the high mass $m_{1}$ and low mass $m_{2}$ neutron stars in a binary, respectively. The curves are corresponding to the EoS with different dark matter density and obtained by varying $m_{1}$ and $m_{2}$ independently. $m_{1}$ has been taken in the range $1.365<m_{1} / M_{\odot}<1.60$ and the range of the $m_{2}$ is determined by keeping the chirp mass $\mathcal{M}_{c}$ fixed at $1.188 M_{\odot}$. The dashed and the dot lines represent, respectively, the $90 \%$ and $50 \%$ confidence limits of the combined dimensionless tidal deformability obtained from the GW170817 for the low spin prior. One can see from this plot that EoS given by the NL3 parametrization without dark matter component can be excluded at $90 \%$ confidence level using the upper bound on tidal deformability of a binary system. However if we consider dark matter component in neutron stars, then NL3 parametrized EoS comes within the 90\% confidence level. Hence a small component of dark matter inside a neutron 
star can revive well-known EoS, which otherwise might be excluded by the GW170817 observation.

\section{CONCLUSIONS}

We have confronted the neutron star equation of state in the presence of dark matter component using the gravitational wave constraint from the binary star merger. We have shown that for a uniformly distributed dark matter inside neutron star, the EoS becomes softer which eventually produces lower NS mass with increasing dark matter density. We have taken the Walecka model with NL3 parametrization in the nuclear matter sector. The Walecka model with NL3 parametrization without dark matter admixture gives rise to a maximum mass of NS $\sim 2.8 M_{\odot}$. By increasing dark matter density (Fermi momentum) inside neutron star reduces the value of maximum mass. The value of the maximum mass of neutron star changes from $2.8 M_{\odot}$ to $2.1 M_{\odot}$ by increasing dark matter Fermi momentum from $0.0 \mathrm{GeV}$ to $0.06 \mathrm{GeV}$. One of the striking results of our analysis is that the stiffer equation of states such as relativistic mean field model (Walecka model) with NL3 parametrization is ruled out at 90\% C.L. using the GW170817 observation. However, in the presence of dark matter this constraint can be evaded and NL3 parametrization can be brought within 90\% C.L.

\section{ACKNOWLEDGMENTS}

T. M. acknowledges the hospitality provided by Physical Research Laboratory (PRL), Ahmedabad, India, during his visit, where the work has been initiated. T. M is also grateful to the Science and Engineering Research Board (SERB), Govt. of India for the international travel support (ITS/2018/002448). We would like to thank Prof. Hiranmaya Mishra for constant encouragement and support.
[1] J. M. Lattimer, Annu. Rev. Nucl. Part. Sci. 62, 485 (2012).

[2] J. D. Walecka, Ann. Phys. (N.Y.) 83, 491 (1974).

[3] B. D. Serot and J. D. Walecka, Int. J. Mod. Phys. E 06, 515 (1997).

[4] J. Boguta and A. R. Bodmer, Nucl. Phys. A292, 413 (1977); J. Boguta and H. Stoecker, Phys. Lett. 120B, 289 (1983);

J. Boguta, Phys. Lett. 106B, 255 (1981).

[5] Y. K. Gambhir, P. Ring, and A. Thimet, Ann. Phys. (N.Y.) 198, 132 (1990).

[6] P. Ring, Prog. Part. Nucl. Phys. 37, 193 (1996).

[7] M. Dutra, O. Lourenço, S. S. Avancini, B. V. Carlson, A. Delfino, D. P. Menezes, C. Providência, S. Typel, and J. R. Stone, Phys. Rev. C 90, 055203 (2014).

[8] G. Baym, C. Pethick, D. Pines, and M. Ruderman, Nature (London) 224, 872 (1969).

[9] W. C. G. Ho, C. M. Espinoza, D. Antonopoulou, and N. Andersson, J. Phys. Soc. Jpn. Conf. Proc. 14, 010805 (2017).

[10] F. Weber, J. Phys. G 25, R195 (1999).

[11] M. Prakash, J. Phys. G 34, S253 (2007).

[12] F. Weber, M. Meixner, P. Negreiros, and M. Malheiro, Int. J. Mod. Phys. E 16, 1165 (2007).

[13] F. Weber, O. Hamil, K. Mimura, and R. Negreiros, Int. J. Mod. Phys. D 19, 1427 (2010).

[14] B. P. Abbott et al., Phys. Rev. Lett. 119, 161101 (2017).

[15] B. P. Abbott et al., Phys. Rev. Lett. 121, 161101 (2018).

[16] T. Hinderer, Astrophys. J. 677, 1216 (2008).

[17] T. Hinderer, B. D. Lackey, R. N. Lang, and J. S. Read, Phys. Rev. D 81, 123016 (2010).

[18] S. Postnikov, M. Prakash, and J. M. Lattimer, Phys. Rev. D 82, 024016 (2010).
[19] K. Yagi and N. Yunes, Science 341, 365 (2013); Phys. Rev. D 88, 023009 (2013).

[20] K. Yagi, L. C. Stein, G. Pappas, N. Yunes, and T. A. Apostolatos, Phys. Rev. D 90, 063010 (2014).

[21] L. Rezzolla, E. R. Most, and L. R. Weih, Astrophys. J. 852, L25 (2018).

[22] L. Rezzolla, E. R. Most, L. R. Weih, and J. SchaffnerBielich, Phys. Rev. Lett. 120, 261103 (2018).

[23] M. Bauer and T. Plehn, arXiv:1705.01987.

[24] N. Raj, P. Tanedo, and H. B. Yu, Phys. Rev. D 97, 043006 (2018).

[25] I. Goldman and S. Nussinov, Phys. Rev. D 40, 3221 (1989).

[26] A. Gould, B. T. Draine, R. W. Romani, and S. Nussinov, Phys. Lett. B 238, 337 (1990).

[27] C. Kouvaris, Phys. Rev. D 77, 023006 (2008).

[28] C. Kouvaris and P. Tinyakov, Phys. Rev. D 82, 063531 (2010).

[29] A. de Lavallaz and M. Fairbairn, Phys. Rev. D 81, 123521 (2010).

[30] T. Guver, A. E. Erkoca, M. H. Reno, and I. Sarcevic, J. Cosmol. Astropart. Phys. 05 (2014) 013.

[31] Yi-zhong Fan, Rui-zhi Yang, and J. Chang, arXiv:1204.2564.

[32] J. Ellis, G. Hutsi, K. Kannike, L. Marzola, M. Raidal, and V. Vaskonen, Phys. Rev. D 97, 123007 (2018).

[33] J. Ellis, A. Hektor, G. Hutsi, K. Kannike, L. Marzola, M. Raidal, and V. Vaskonen, Phys. Lett. B 781, 607 (2018).

[34] Q.-F. Xiang, W.-Z. Jiang, D.-R. Zhang, and R.-Y. Yang, Phys. Rev. C 89, 025803 (2014).

[35] G. Panotopoulos and I. Lopes, Phys. Rev. D 96, 083004 (2017).

[36] K. C. Chung, C. S. Wang, A. J. Santiago, and J. W. Zhang, Eur. Phys. J. A 11, 137 (2001). 
[37] G. A. Lalazissis, J. Konig, and P. Ring, Phys. Rev. C 55, 540 (1997).

[38] J. Boguta, Phys. Lett. 106B, 245 (1981).

[39] Advances in Nuclear Physics, edited by J. W. Negele and E. Vogt (Plenum, New York, 1986), Vol. 16.

[40] I. Goldman and S. Nussinov, Phys. Rev. D 40, 3221 (1989).

[41] L. Brayeur and P. Tinyakov, Phys. Rev. Lett. 109, 061301 (2012).

[42] S. P. Martin, Adv. Ser. Dir. High Energy Phys. 21, 1 (2010); B. Murakami and J. D. Wells, Phys. Rev. D 64, 015001 (2001).

[43] J. M. Cline, K. Kainulainen, P. Scott, and C. Weniger, Phys. Rev. D 88, 055025 (2013); 92, 039906(E) (2015).

[44] C. Frederico Pascoal da Silva (LUX Collaboration), arXiv: 1710.03572.

[45] E. Aprile et al. (Xenon Collaboration), Phys. Rev. Lett. 119, 181301 (2017).
[46] J. R. Oppenheimer and G. M. Volkoff, Phys. Rev. 55, 374 (1939).

[47] G. Baym, C. Pethick, and P. Sutherland, Astrophys. J. 170, 299 (1971).

[48] J. Carriere, C. J. Horowitz, and J. Piekarewicz, Astrophys. J. 593, 463 (2003).

[49] T. Malik, K. Banerjee, T. K. Jha, and B. K. Agrawal, Phys. Rev. C 96, 035803 (2017).

[50] T. Malik, N. Alam, M. Fortin, C. Providencia, B. K. Agrawal, T. K. Jha, B. Kumar, and S. K. Patra, Phys. Rev. C 98, 035804 (2018).

[51] C. Raithel, F. Ozel, and D. Psaltis, Astrophys. J. 857, L23 (2018).

[52] S. De, D. Finstad, J. M. Lattimer, D. A. Brown, E. Berger, and C. M. Biwer, Phys. Rev. Lett. 121, 091102 (2018); 121, 259902(E) (2018).

[53] D. Radice, A. Perego, F. Zappa, and S. Bernuzzi, Astrophys. J. Lett. 852, L29 (2018). 